\title{
Development of an animal model: A key role in investigational research
}

\author{
I. Schade, M. Knaut, K. Plötze and K. Matschke* \\ Heart Centre Dresden, Technical University, Dresden, Germany
}

\section{Background}

In order to investigate the properties of certain materials a biological model is essential before establishing a clinical trial [1]. Therefore development of an animal model can be a major part of experimental work.

In this study we developed animal models for testing a material later to be used in vascular or heart surgery. Threads, arterial patches and valve conduits were supposed to be implanted. Goal of the study was to test the material up to $60 \mathrm{~d}$ without any systemic anticoagulation to evaluate its antithrombotic ability. Therefore the extent of the surgical procedure was crucial.

In a first step Sprague Dawley rats with a weight of $250 \mathrm{~g}$ were used. A thread of the material was introduced into the abdominal aorta. Due to severe bleeding complications and secondary mortality this model had to be revised. Since the material was rigid and stiff and could not be changed another animal had to be established. In a second trial rabbits were used with no surgical problems. For implantation of the valve conduits a larger model, domestic pigs, was used. This model had the advantage of being very similar regarding the blood, the anatomy and the volume of the heart. Also the coagulation pathway is comparable to humans.

\section{Material and methods}

For all thread and patch implantations New Zealand White rabbits with a weight of 2.5-3.0 kg were used. Following the anaesthesia by Xylacin (2\%) and Ketamin (5\%) the thread implantation was performed in Seldinger technique with outside fixation. For the patch implantation a shunt (used in Off Pump surgery) was introduced (Fig. 1A).

In the pig model implantation of a modified tri-leaflet valve into the descending aorta without circulatory support was planned. Surgery was performed in general anesthesia, with intermittent one side ventilation during left side thoracotomy (7./8. intercostal space). Surgery was done in cross clamp technique (Fig. 2A). The valve graft was interposed between the two stumps of the descending aorta with

\footnotetext{
${ }^{*}$ Corresponding author: Dr. K. Matschke, PD, Heart Centre Dresden, Technical University, Fetscherstraße 76, D-01307 Dresden, Germany. E-mail: k.matschke@ herzzentrum-dresden.com.
} 

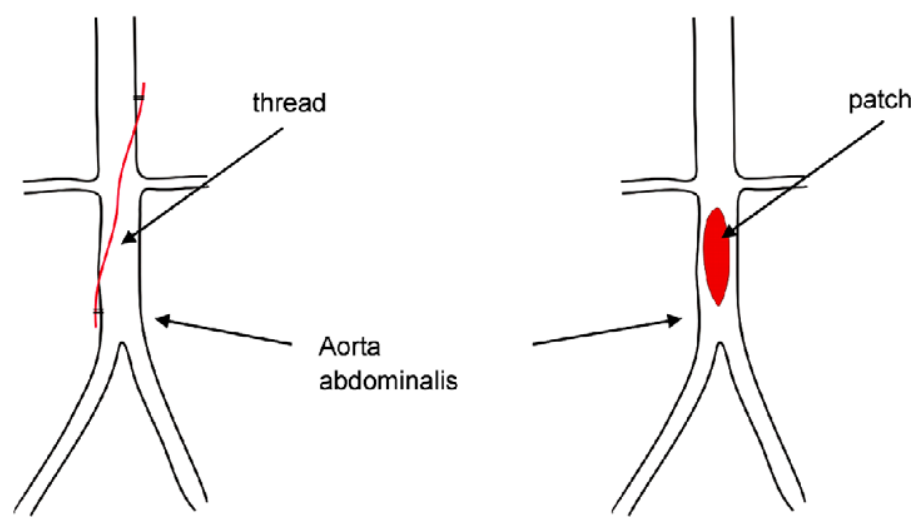

Fig. 1. Sketch of the rabbit model (left: thread-implantation; right: patch-implantation).

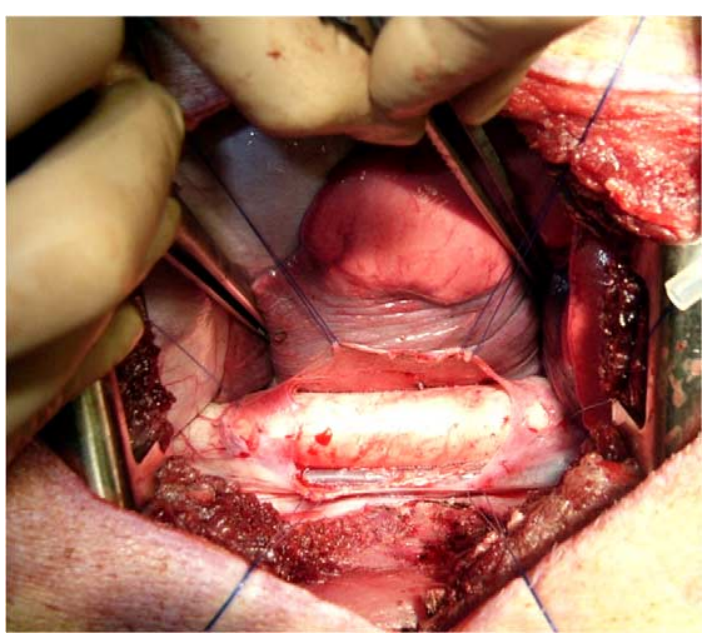

A

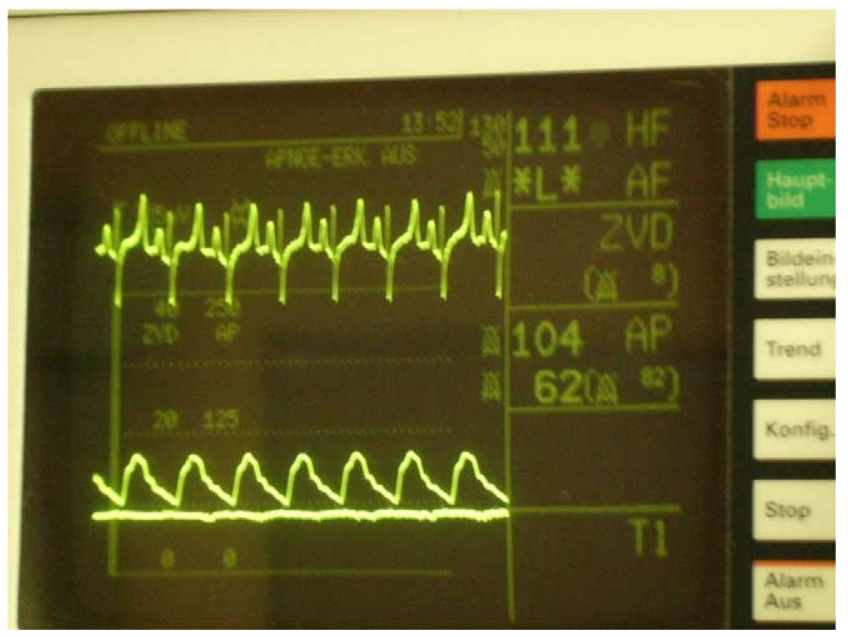

B

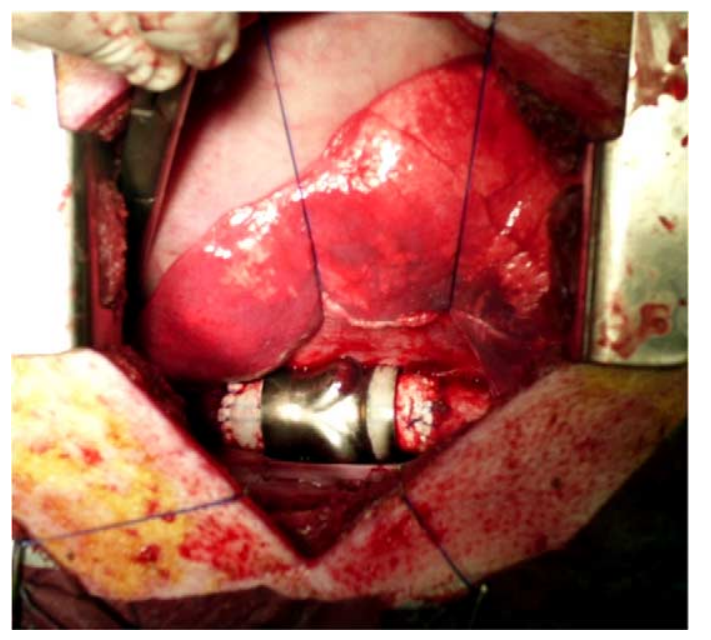

C

Fig. 2. Preparation of the thoracic aorta for valve implantation. Monitoring during operation (ECG; CVP; AP). Implantation of a valve conduit in the descending aorta. 
5/0 Prolene sutures (Fig. 2C). Main problems encountered were hemodynamic instability and postoperative neurological deficits. Therefore the surgical approach was changed to using also a shunt (like a Gott-Shunt) (Fig. 2C).

\section{Results}

\subsection{Rabbit models}

In this model no bleeding or other complications occurred over the whole study period. 32 rabbits were operated on for thread-implantations and reexplored after 14 days (Fig. 3A/B). 18 rabbits were operated on for patch-implantation and reexplored after 4 weeks (Fig. 4A/B). All animals survived without any anticoagulation.

\subsection{Pig model}

Main problems of this model were hemodynamic instability and postoperative neurological deficits, like paraplegia of the lower extremities. In the literature the risk of paraplegia in swine is described at 15-20\%, because of the low tolerance of spinal cord ischemia. Thus we changed the surgical approach and introduced a shunt similar to a Goot-Shunt used in heart surgery. After that the postoperative course was uneventful in all cases with a drop of the paraplegia rate to $7.7 \%$. This is comparable to real life surgery of the descending aorta in humans and in animal surgery. All swine were doing well after 4 weeks.

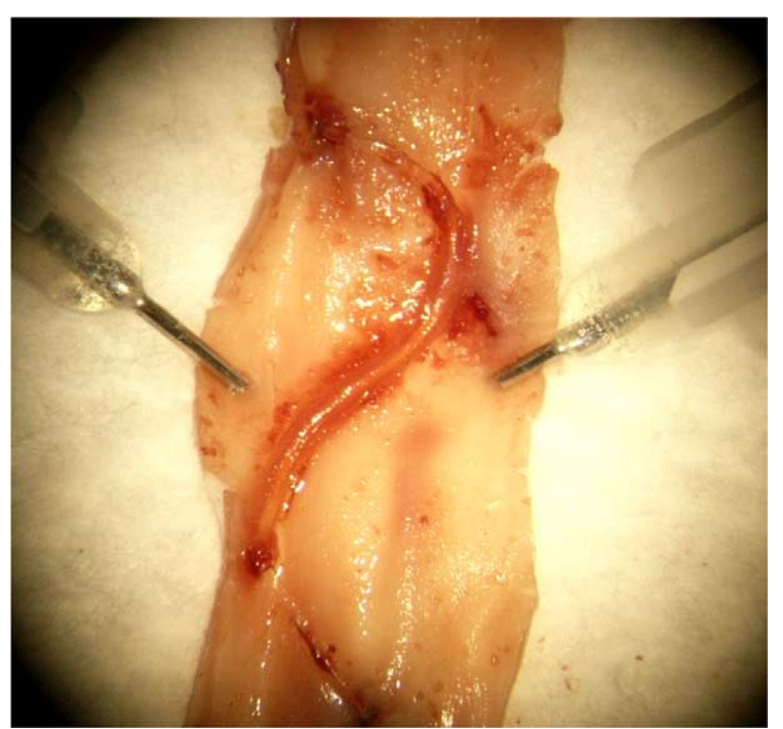

A

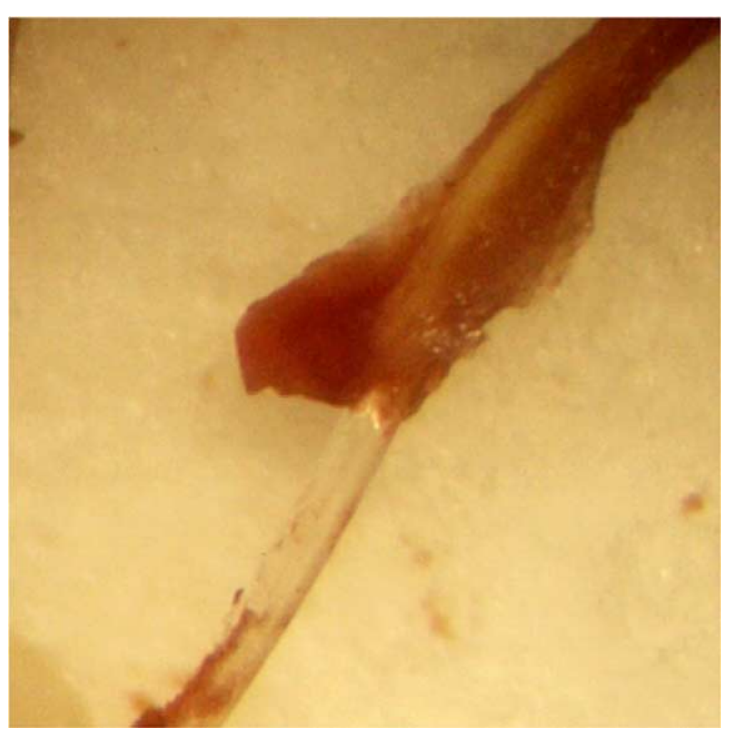

B

Fig. 3. Result after thread-implantation (after 2 weeks; fibrous tissue material around the thread). Single thread with fibrous material. 


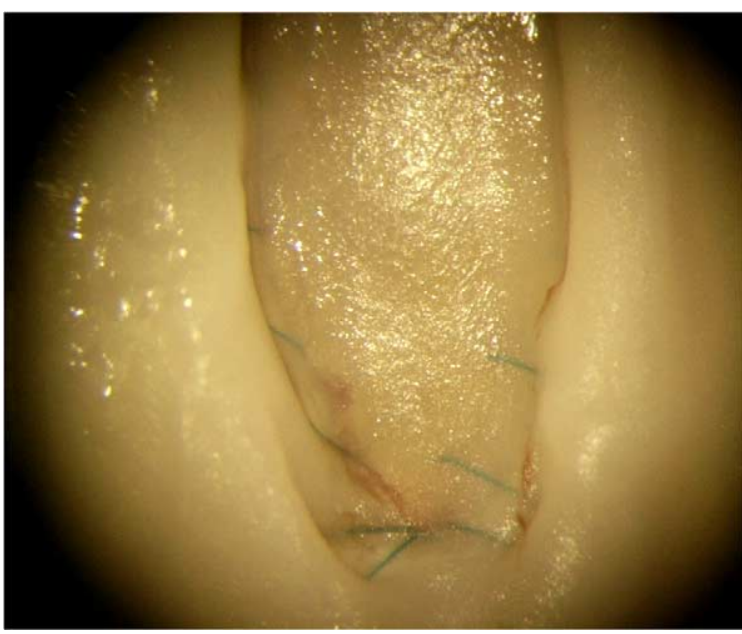

A

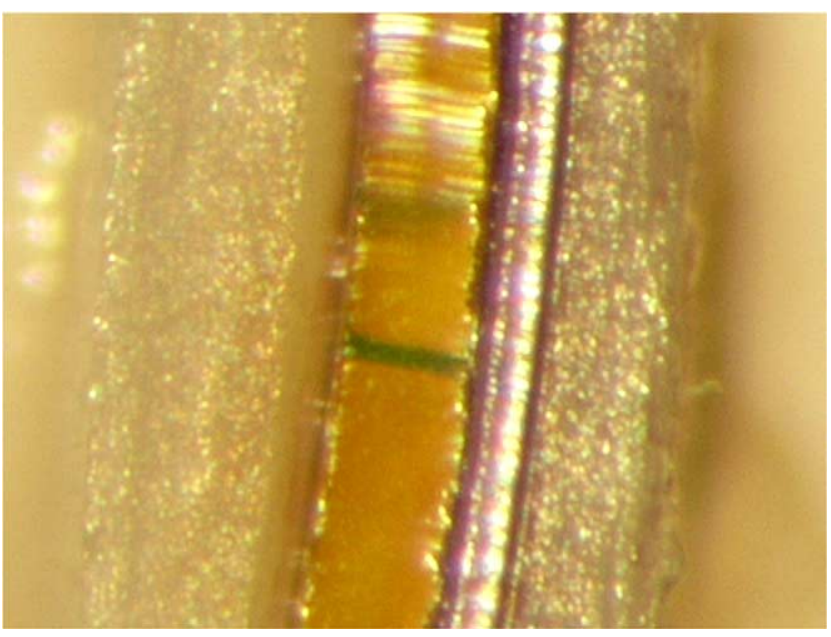

B

Fig. 4. Result after patch-implantation (after 4 weeks; intraluminal: the patch covered by a cellular layer). Result after patch-implantation (patch covered by a cellular layer; left to right: cellular layer - patch - outside layer).

\section{Summary}

The rabbit model is sufficient for testing the described material in a sufficient blood vessel. The pig is a well established model for all surgical implications. Even in difficult situations as described a change in surgical approach, in this case by using a method common in heart surgery, leads to very desirable results. The condition of no anticoagulation could be achieved.

\section{Conclusion}

- Different animal models allow reproducible results with a good survival and no major complications.

- Due to their efficacy those animal models can be used in research endeavors concerning blood and its coagulation parameters over a longer time.

- All models are suitable for experimental procedures without the necessity of circulatory support.

\section{References}

[1] D. Rickert, M.O. Scheithauer, S. Coskun, S. Kelch, A. Lendlein and R.P. Franke, The influence of a multifunctional, polymeric biomaterial on the concentration of acute phase proteins in an animal model, Clin. Hemorheol. Microcirc. 36 (2007), 301-311. 